TRANSACTIONS OF THE

AMERICAN MATHEMATICAL SOCIETY

Volume 359, Number 1, January 2007, Pages 77-89

S 0002-9947(06)03930-4

Article electronically published on July 20, 2006

\title{
PERTURBATION OF SPECTRA AND SPECTRAL SUBSPACES
}

\author{
VADIM KOSTRYKIN, K. A. MAKAROV, AND A. K. MOTOVILOV \\ Dedicated to Volker Enss on the occasion of his 60th birthday
}

\begin{abstract}
We consider the problem of variation of spectral subspaces for linear self-adjoint operators with emphasis on the case of off-diagonal perturbations. We prove a number of new optimal results on the shift of the spectrum and obtain (sharp) estimates on the norm of the difference of two spectral projections associated with isolated parts of the spectrum of the perturbed and unperturbed operators, respectively.
\end{abstract}

\section{INTRODUCTION}

It is well known (see, e.g., [12]) that if $A$ and $V$ are bounded self-adjoint operators on a separable Hilbert space $\mathfrak{H}$, then the spectrum of the operator $B=A+V$ is confined in the closed $\|V\|$-neighborhood $\mathcal{U}_{\|V\|}(\operatorname{spec}(A))$ of the spectrum of $A$,

$$
\operatorname{spec}(B) \subset \mathcal{U}_{\|V\|}(\operatorname{spec}(A)) .
$$

In particular, if the spectrum $\operatorname{spec}(A)$ consists of two isolated parts $\sigma$ and $\Sigma=$ $\operatorname{spec}(A) \backslash \sigma$ such that $\operatorname{dist}(\sigma, \Sigma)=d>0$, under the hypothesis

$$
\|V\|<\frac{d}{2}
$$

the perturbation $V$ does not close the gaps in the spectrum of $A$ separating the sets $\sigma$ and $\Sigma$ and hence the spectrum of $B$ also has two separated components. Clearly, condition (2) is sharp in the sense that if $\|V\| \geq d / 2$, the perturbed operator $A+V$ may not have separated parts of the spectrum at all.

One of fundamental problems of the perturbation theory is to study the variation of the spectral subspace associated with the isolated part $\sigma$ of the spectrum of $A$ under the perturbation. A still unsolved problem is to give an answer to the following question: Is it true or not that under the hypothesis (2)

$$
\left\|\mathrm{E}_{A}(\sigma)-\mathrm{E}_{B}\left(\mathcal{O}_{d / 2}(\sigma)\right)\right\|<1 ?
$$

Here $\mathrm{E}_{T}(\Delta)$ denotes the spectral projection for the self-adjoint operator $T$ corresponding to a Borel set $\Delta$ and $\mathcal{O}_{d / 2}(\sigma)$ is the open $d / 2$-neighborhood of the set $\sigma$ (see [13] for a partially affirmative answer to this question).

In the present paper we treat the case where the perturbation $V$ is off-diagonal with respect to the direct sum of spectral subspaces associated with the sets $\sigma$ and $\Sigma=\operatorname{spec}(A) \backslash \sigma$ in the sense that $\mathrm{E}_{A}(\sigma) V \mathrm{E}_{A}(\sigma)=\mathrm{E}_{A}(\Sigma) V \mathrm{E}_{A}(\Sigma)=0$. We address the following general question for the off-diagonal perturbations.

Received by the editors September 23, 2004.

2000 Mathematics Subject Classification. Primary 47A15, 47A55; Secondary 47B15. 
(i) What is an optimal requirement on the norm of the perturbation $V$ that guarantees that $V$ does not close the gaps in the spectrum of $A$ separating the sets $\sigma$ and $\Sigma$ ?

Unlike the case of general perturbations, in the off-diagonal case the answer depends upon the mutual disposition of the isolated parts $\sigma$ and $\Sigma$ of the spectrum of the operator $A$. Leaving apart the well-known case where the sets $\sigma$ and $\Sigma$ are subordinated (see [3, , 9], [10, [15]) we focus on two cases:

Case I: the sets $\sigma$ and $\Sigma$ are separated.

Case II: the set $\sigma$ and the convex hull of $\Sigma$ (or vice versa) are separated.

We give a complete solution to the problem (i) and show that the corresponding optimal requirements are: $\|V\|<\sqrt{3} / 2 d$ in Case I and $\|V\|<\sqrt{2} d$ in Case II, respectively.

We also address the following question of perturbation theory for spectral subspaces.

(ii) What can be said about variation of the spectral subspace associated with the isolated part $\sigma$ of the spectrum of $A$ under the off-diagonal perturbations satisfying the optimal requirements above?

We conjecture that in Case I the inequality $\|V\|<\sqrt{3} / 2 d$ is sufficient for the difference of the projections $\mathrm{E}_{A}(\sigma)-\mathrm{E}_{B}\left(\mathcal{O}_{d / 2}(\sigma)\right)$ to be a strict contraction. We also prove that in Case II the optimal "gap-nonclosing" requirement $\|V\|<\sqrt{2} d$ guarantees that $\left\|\mathrm{E}_{A}(\sigma)-\mathrm{E}_{B}\left(\mathcal{O}_{d}(\sigma)\right)\right\|<1$.

Main results. Let

$$
\delta_{V}=\|V\| \tan \left(\frac{1}{2} \arctan \frac{2\|V\|}{d}\right) .
$$

Our first principal result is as follows.

Theorem 1. Suppose that the self-adjoint bounded perturbation $V$ is off-diagonal with respect to the decomposition $\mathcal{H}=\operatorname{RanE}_{A}(\sigma) \oplus \operatorname{RanE}_{A}(\Sigma)$. If

$$
\|V\|<\frac{\sqrt{3}}{2} d
$$

or, which is the same, $\delta_{V}<d / 2$, then

(i) the spectrum of $B$ in the open $d / 2$-neighborhood $\mathcal{O}_{d / 2}(\sigma)$ of the set $\sigma$ is separated from the remainder of the spectrum of $B$. Moreover,

$$
\operatorname{spec}(B) \cap \mathcal{O}_{d / 2}(\sigma)=\operatorname{spec}(B) \cap \mathcal{U}_{\delta_{V}}(\sigma)
$$

is a nonempty closed set;

(ii) if in addition $\|V\|<c_{\pi} d$ with $c_{\pi}=\frac{3 \pi-\sqrt{\pi^{2}+32}}{\pi^{2}-4}=0.503288 \ldots$, then

$$
\left\|\mathrm{E}_{A}(\sigma)-\mathrm{E}_{B}\left(\mathcal{O}_{d / 2}(\sigma)\right)\right\| \leq \frac{\pi}{2} \frac{\|V\|}{d-\delta_{V}}<1 .
$$

This result is sharp in the sense that if the norm bound $\|V\|<\frac{\sqrt{3}}{2} d$ is violated, then the set $\operatorname{spec}(B) \cap \mathcal{O}_{\delta_{V}}(\sigma)$ may be either empty or non-closed (see Example 1.5 below). Theorem 1 implies that the best possible constant $c$ in the inequality $\|V\|<c d$ implying $\left\|\mathrm{E}_{A}(\sigma)-\mathrm{E}_{B}\left(\mathcal{O}_{d / 2}(\sigma)\right)\right\|<1$ satisfies the two-sided estimate

$$
c_{\pi} \leq c \leq \frac{\sqrt{3}}{2}
$$


improving the previously known bounds $1 / \pi \leq c \leq \sqrt{2}$ [4] and $\frac{2}{2+\pi} \leq c$ [13].

If the convex hull $\mathcal{K}(\sigma)$ of the set $\sigma$ does not intersect the remainder $\Sigma$ of the spectrum of $A$ we face a new phenomenon which does not have an analog in the case of general perturbations. That is, the spectrum of the component $\Sigma$ may not "leak out" into the open $d$-neighborhood of the set $\sigma$, provided that $\|V\|<\sqrt{2} d$ and the perturbation $V$ is off-diagonal.

We give a complete solution of the problem in this case and our second principal result is as follows.

Theorem 2. Suppose that the self-adjoint bounded perturbation $V$ is off-diagonal with respect to the decomposition $\mathcal{H}=\operatorname{RanE}_{A}(\sigma) \oplus \operatorname{RanE}_{A}(\Sigma)$. If $\mathcal{K}(\sigma) \cap \Sigma=\emptyset$ and

$$
\|V\|<\sqrt{2} d
$$

or, which is the same, $\delta_{V}<d$, then

(i) the spectrum of $B$ in the open d-neighborhood $\mathcal{O}_{d}(\sigma)$ of the set $\sigma$ is separated from the remainder of the spectrum of $B$. Moreover,

$$
\operatorname{spec}(B) \cap \mathcal{O}_{d}(\sigma)=\operatorname{spec}(B) \cap \mathcal{U}_{\delta_{V}}(\sigma) \quad \text { is a nonempty closed set and }
$$

(ii)

$$
\left\|\mathrm{E}_{A}(\sigma)-\mathrm{E}_{B}\left(\mathcal{O}_{d}(\sigma)\right)\right\| \leq \sin \left(\arctan \frac{\|V\|}{d-\delta_{V}}\right)<1 .
$$

This result is sharp in the following sense. If the norm bound $\|V\|<\sqrt{2} d$ is violated, then the set $\operatorname{spec}(B) \cap \mathcal{O}_{\delta_{V}}(\sigma)$ may be either empty or non-closed (see Example 1.6 below). Moreover, the best possible constant $c$ in inequality $\|V\|<c d$ implying $\left\|\mathrm{E}_{A}(\sigma)-\mathrm{E}_{B}\left(\mathcal{O}_{d}(\sigma)\right)\right\|<1$ is $c=\sqrt{2}$. Note that the size of the neighborhood in question is as twice as big as that in Theorem 1 .

As we have already mentioned, the case where the sets $\sigma$ and $\Sigma$ are subordinated is well understood and the following is known (see [2, 9, [10, 15]).

Theorem 3. Suppose that the self-adjoint bounded perturbation $V$ is off-diagonal with respect to the decomposition $\mathcal{H}=\operatorname{RanE}_{A}(\sigma) \oplus \operatorname{RanE}_{A}(\Sigma)$. If the sets $\sigma$ and $\Sigma$ are subordinated and, for definiteness, $\sup \sigma<\inf \Sigma$, then

(i) the spectrum of the operator $B$ does not intersect the open interval $(\sup \sigma$, $\inf \Sigma$ ) and

$$
\left\|\mathrm{E}_{A}(\sigma)-\mathrm{E}_{B}((-\infty, \sup \sigma])\right\| \leq \sin \left(\frac{1}{2} \arctan \frac{2\|V\|}{d}\right)<\frac{\sqrt{2}}{2} .
$$

In particular, the spectrum of the perturbed operator $B$ always has two subordinated components and the perturbation $V$ does not close the gap $(\sup \sigma, \inf \Sigma)$ in the spectrum of $A$ (no requirements on the norm of $V$ are needed). An analog of Theorem 3 for the case without gap, that is, for $\sup \sigma \leq \inf \Sigma$ or $\sup \Sigma \leq \inf \sigma$ is also known (see [3] and [15]).

A few words about notations. $\operatorname{By} \operatorname{spec}(A)$ we denote the spectrum of a bounded self-adjoint operator $A$ and $\inf A(\sup A)$ denotes the infimum (supremum) of the set $\operatorname{spec}(A)$. The spectral projection of $A$ associated with a Borel set $\Delta \subset \mathbb{R}$ is denoted by $\mathrm{E}_{A}(\Delta)$ and the resolvent set of $A$ is denoted by $\rho(A)$. We use the symbol $\mathcal{O}$ for open sets while the symbol $\mathcal{U}$ is usually associated with closed neighborhoods. If not explicitly stated otherwise, for an arbitrary orthogonal projection $P$ the symbol $P^{\perp}$ 
denotes the orthogonal projection onto the orthogonal complement of the subspace $\operatorname{Ran} P$ in $\mathcal{H}$, i.e., $P^{\perp}=I-P$.

\section{Perturbation of spectra}

We start this section by presenting a fairly simple but general result which provides optimal lower and upper bounds on the shift of the spectrum of a bounded self-adjoint operator under a perturbation which is off-diagonal with respect to the given orthogonal decomposition of the Hilbert space reducing the unperturbed operator.

Lemma 1.1. Let $A$ and $V$ be bounded self-adjoint operators on a Hilbert space $\mathcal{H}, B=A+V$, and $P$ an orthogonal projection commuting with A. Assume, in addition, that

$$
P V P=P^{\perp} V P^{\perp}=0 .
$$

Denote by $A_{0}$ and $A_{1}$ the parts of $A$ associated with its invariant subspaces $\operatorname{Ran} P$ and $\operatorname{Ran} P^{\perp}$, respectively.

Then

$$
\inf A-\delta_{V}^{\ell} \leq \inf B \leq \inf A
$$

and

$$
\sup A \leq \sup B \leq \sup A+\delta_{V}^{r},
$$

where

$$
\begin{aligned}
& \delta_{V}^{\ell}=\|V\| \tan \left(\frac{1}{2} \arctan \frac{2\|V\|}{\left|\inf A_{1}-\inf A_{0}\right|}\right), \\
& \delta_{V}^{r}=\|V\| \tan \left(\frac{1}{2} \arctan \frac{2\|V\|}{\left|\sup A_{1}-\sup A_{0}\right|}\right)
\end{aligned}
$$

with a natural convention that $\arctan (+\infty)=\pi / 2$ in the case where $\inf A_{1}=\inf A_{0}$ and/or $\sup A_{1}=\sup A_{0}$.

Proof. Denote by $W^{2}(B)$ (cf. [16]) the quadratic numerical range of the operator $B$ with respect to the decomposition $\mathcal{H}=\operatorname{Ran} P \oplus \operatorname{Ran} P^{\perp}$,

$$
W^{2}(B)=\bigcup_{\substack{\|f\|=\|\|\|\|=1 \\
f \in \operatorname{Ran} P \\
g \in \operatorname{Ran} P^{\perp}}} \operatorname{spec}\left(\begin{array}{ll}
(f, B f) & (f, B g) \\
(g, B f) & (g, B g)
\end{array}\right) .
$$

For $f$ and $g$ as above taking into account (1.1) yields

$$
\left(\begin{array}{ll}
(f, B f) & (f, B g) \\
(g, B f) & (g, B g)
\end{array}\right)=\left(\begin{array}{ll}
(f, A f) & (f, V g) \\
(g, V f) & (g, A g)
\end{array}\right)=\left(\begin{array}{cc}
a_{0} & v \\
v^{*} & a_{1}
\end{array}\right)
$$

where we have introduced the notations $a_{0}=\left(A_{0} f, f\right), a_{1}=\left(g, A_{1} g\right)$, and $v=$ $(f, V g)$. The matrix $\left(\begin{array}{cc}a_{0} & v \\ v^{*} & a_{1}\end{array}\right)$ has two eigenvalues $\lambda$ and $\mu$ given by

and

$$
\lambda=\min \left\{a_{0}, a_{1}\right\}-|v| \tan \left(\frac{1}{2} \arctan \frac{2|v|}{\left|a_{1}-a_{0}\right|}\right)
$$

$$
\mu=\max \left\{a_{0}, a_{1}\right\}+|v| \tan \left(\frac{1}{2} \arctan \frac{2|v|}{\left|a_{1}-a_{0}\right|}\right) .
$$


Clearly the eigenvalues $\lambda$ and $\mu$ satisfy the inequalities

$$
\inf A-\delta_{V}^{\ell} \leq \lambda \leq \min \left\{a_{0}, a_{1}\right\}
$$

and

$$
\max \left\{a_{0}, a_{1}\right\} \leq \mu \leq \sup A+\delta_{V}^{r} .
$$

Since the quadratic numerical range $W^{2}(B)$ contains the spectrum of $B$ while $\inf W^{2}(B)=\inf B$ and $\sup W^{2}(B)=\sup B$ (see [16]), estimates (1.4) and (1.5) prove the assertion.

Given the result of Lemma 1.1, now the proof of Theorem1(i) and Theorem 2(i) is straightforward.

For notational setup introduce the following hypothesis.

Hypothesis 1.2. Assume that $A$ and $V$ are bounded self-adjoint operators on a separable Hilbert space $\mathfrak{H}$. Suppose that the spectrum of $A$ has a part $\sigma$ separated from the remainder of the spectrum $\Sigma$ in the sense that

$$
\operatorname{spec}(A)=\sigma \cup \Sigma
$$

and

$$
\operatorname{dist}(\sigma, \Sigma)=d>0
$$

Assume, in addition, that $V$ is off-diagonal with respect to the decomposition $\mathcal{H}=$ $\operatorname{RanE}_{A}(\sigma) \oplus \operatorname{RanE}_{A}(\Sigma)$.

Theorem 1.3. Assume Hypothesis [1.2, Then

(i) The spectrum of the operator $B$ is contained in the closed $\delta_{V}$-neighborhood $\mathcal{U}_{\delta_{V}}(\operatorname{spec}(A))$ of the spectrum of $A$

$$
\operatorname{spec}(B) \subset \mathcal{U}_{\delta_{V}}(\operatorname{spec}(A)),
$$

where

$$
\delta_{V}=\|V\| \tan \left(\frac{1}{2} \arctan \frac{2\|V\|}{d}\right)
$$

(ii) If

$$
\|V\|<\frac{\sqrt{3}}{2} d
$$

(or, which is the same, $\delta_{V}<d / 2$ ), then the spectrum of $B$ in the open $d / 2$ neighborhood $\mathcal{O}_{d / 2}(\sigma)$ of the set $\sigma$ is separated from the remainder of the spectrum of $B$. That is,

$$
\operatorname{spec}(B) \cap \mathcal{O}_{d / 2}(\sigma)=\operatorname{spec}(B) \cap \mathcal{U}_{\delta_{V}}(\sigma) \quad \text { is a nonempty closed set. }
$$

(iii) If $\mathcal{K}(\sigma) \cap \Sigma=\emptyset$ and

$$
\|V\|<\sqrt{2} d
$$

(or, which is the same, $\delta_{V}<d$ ), then the spectrum of $B$ in the open $d$-neighborhood $\mathcal{O}_{d}(\sigma)$ of the set $\sigma$ is separated from the remainder of the spectrum of $B$. That is,

$$
\operatorname{spec}(B) \cap \mathcal{O}_{d}(\sigma)=\operatorname{spec}(B) \cap \mathcal{U}_{\delta_{V}}(\sigma) \quad \text { is a nonempty closed set. }
$$


Proof. (i) Take a $\lambda \in \mathbb{R}$ such that

$$
\operatorname{dist}(\lambda, \operatorname{spec} A)>\delta_{V} .
$$

Denote by $A_{\ell}$ the part of the operator $A$ associated with the $A$-invariant subspace

$$
\mathcal{L}=\operatorname{RanE}_{A}((-\infty, \lambda))
$$

and let $V_{\ell}=\left.\mathrm{E}_{A}((-\infty, \lambda)) V\right|_{\mathcal{L}}$. By Hypothesis 1.2 the operator $V$ is off-diagonal with respect to the decomposition $\mathcal{H}=\mathrm{E}_{A}(\sigma) \mathcal{H} \oplus \mathrm{E}_{A}(\Sigma) \mathcal{H}$, so is $V_{\ell}$ with respect to the decomposition $\mathcal{L}=\mathrm{E}_{A_{\ell}}(\sigma \cap(-\infty, \lambda)) \mathcal{L} \oplus \mathrm{E}_{A_{\ell}}(\Sigma \cap(-\infty, \lambda)) \mathcal{L}$.

Applying Lemma 1.1 yields

$$
\sup \left(A_{\ell}+V_{\ell}\right) \leq \sup A_{\ell}+\delta_{V} .
$$

Similarly introducing $A_{r}$ as the part of the operator $A$ associated with the $A$ invariant subspace $\mathcal{R}=\operatorname{RanE}_{A}((\lambda, \infty))$ and $V_{r}$ as $\left.\mathrm{E}_{A}((\lambda, \infty)) V\right|_{\mathcal{R}}$ one proves that

$$
\inf \left(A_{r}+V_{r}\right) \geq \inf \left(A_{r}\right)-\delta_{V} .
$$

Combining (1.9), (1.10), and (1.8) proves that

$$
\sup \left(A_{\ell}+V_{\ell}\right)<\lambda<\inf \left(A_{r}+V_{r}\right) .
$$

Clearly the operator $B$ can be represented as follows

$$
B=\operatorname{diag}\left\{A_{\ell}+V_{\ell}, A_{r}+V_{r}\right\}+W,
$$

where $W$ is given by

$$
W=V-\mathrm{E}_{A}((-\infty, \lambda)) V \mathrm{E}_{A}((-\infty, \lambda))-\mathrm{E}_{A}((\lambda, \infty)) V \mathrm{E}_{A}((\lambda, \infty))
$$

and $\operatorname{diag}\left\{A_{\ell}+V_{\ell}, A_{r}+V_{r}\right\}$ is a diagonal $2 \times 2$ operator matrix with respect to the decomposition $\mathcal{H}=\mathcal{L} \oplus \mathcal{R}$. Since $W$ is off-diagonal with respect to $\mathcal{H}=\mathcal{L} \oplus \mathcal{R}$, and the spectra of the diagonal entries $A_{\ell}+V_{\ell}$ and $A_{r}+V_{r}$ are subordinated, the whole interval $\left(\sup \left(A_{\ell}+V_{\ell}\right), \inf \left(A_{r}+V_{r}\right)\right)$ belongs to the resolvent set of $B$ (see, e.g., [10] or [2]), in particular $\lambda$ belongs to the resolvent set of the operator $B$, completing the proof.

Before proving assertions (ii) and (iii) of the theorem, note that the function

$$
f(x)=x \tan \left(\frac{1}{2} \arctan 2 x\right)
$$

is strictly increasing on the positive semi-axis and, moreover, by direct computation one gets

$$
f\left(\frac{\sqrt{3}}{2}\right)=\frac{1}{2} \quad \text { and } \quad f(\sqrt{2})=1 .
$$

In particular,

$$
\text { the inequality }\|V\|<\frac{\sqrt{3}}{2} d \text { implies } \quad \delta_{V}<d / 2
$$

and

$$
\text { the inequality }\|V\|<\sqrt{2} d \text { implies } \delta_{V}<d \text {. }
$$

(ii) The part (ii) is an immediate corollary of the part (i) taking into account (1.11).

(iii) Take

$$
\lambda=\sup \sigma+\delta_{V}
$$


and let $A_{\ell}, A_{r}$, and $V_{\ell}, V_{r}$ be as above. Note that the hypothesis $\mathcal{K}(\sigma) \cap \Sigma=\emptyset$ implies $V_{r}=0$.

Again, as in the proof of (ii) one concludes that

$$
\sup \left(A_{\ell}+V_{\ell}\right) \leq \sup \operatorname{spec}\left(A_{\ell}\right)+\delta_{V}=\sup \sigma+\delta_{V} .
$$

Hypothesis (1.7) implies that $\delta_{V}<d$. Since $V_{r}=0$, the operator $B$ can be represented in the form

$$
B=\operatorname{diag}\left\{A_{\ell}+V_{\ell}, A_{r}\right\}+W
$$

where $W$ is given by

$$
W=V-\mathrm{E}_{A}((-\infty, \lambda)) V \mathrm{E}_{A}((-\infty, \lambda))-\mathrm{E}_{A}((\lambda, \infty)) V \mathrm{E}_{A}((\lambda, \infty))
$$

and $\operatorname{diag}\left\{A_{\ell}+V_{\ell}, A_{r}\right\}$ is a diagonal $2 \times 2$ operator matrix with respect to the decomposition $\mathcal{H}=\mathcal{L} \oplus \mathcal{R}$. Since $W$ is off-diagonal with respect to $\mathcal{H}=\mathcal{L} \oplus \mathcal{R}$, and the spectra of the diagonal entries $A_{\ell}+V_{\ell}$ and $A_{r}$ are subordinated $\left(\delta_{V}<d\right)$, the whole interval $\left(\sup \left(A_{\ell}+V_{\ell}\right), \inf \left(A_{r}\right)\right)$ belongs to the resolvent set of $B$. In particular, the interval $\left(\sup \sigma+\delta_{V}, \sup \sigma+d\right)$ belongs to the resolvent set of the operator $B$, that is,

$$
\left(\sup \sigma+\delta_{V}, \sup \sigma+d\right) \subset \rho(B) .
$$

The proof of the inclusion

$$
\left(\inf \sigma-d, \inf \sigma-\delta_{V}\right) \subset \rho(B)
$$

is analogous.

Remark 1.4. The results (ii) and (iii) are optimal. That is, if the perturbation $V$ is overcritical in the sense that $\|V\| \geq \frac{\sqrt{3}}{2} d($ resp. $\mathcal{K}(\sigma) \cap \Sigma=\emptyset$ and $\|V\| \geq \sqrt{2} d$ ), then the set $\mathcal{O}_{d / 2}(\sigma) \cap \operatorname{spec}(B)\left(\operatorname{resp} . \mathcal{O}_{d}(\sigma) \cap \operatorname{spec}(B)\right)$ may be empty.

The following two examples illustrate the situation.

Example 1.5. Let $\mathcal{H}=\mathbb{C}^{4}$. Introducing the $4 \times 4$ matrices

$$
A=\left(\begin{array}{cccc}
-\frac{3}{2} & 0 & 0 & 0 \\
0 & -\frac{1}{2} & 0 & 0 \\
0 & 0 & \frac{1}{2} & 0 \\
0 & 0 & 0 & \frac{3}{2}
\end{array}\right) \quad \text { and } \quad V=\left(\begin{array}{cccc}
0 & \frac{\sqrt{3}}{2} & 0 & 0 \\
0 & 0 & 0 & \frac{\sqrt{3}}{2} \\
\frac{\sqrt{3}}{2} & 0 & 0 & 0 \\
0 & 0 & \frac{\sqrt{3}}{2} & 0
\end{array}\right)
$$

one easily verifies that the spectrum of the $4 \times 4$ Jacobi matrix

$$
B=\left(\begin{array}{cccc}
-\frac{3}{2} & \frac{\sqrt{3}}{2} & 0 & 0 \\
\frac{\sqrt{3}}{2} & -\frac{1}{2} & 0 & 0 \\
0 & 0 & \frac{1}{2} & \frac{\sqrt{3}}{2} \\
0 & 0 & \frac{\sqrt{3}}{2} & \frac{3}{2}
\end{array}\right)
$$

consists of the three eigenvalues $-2,0$, and 2 , with 0 being an eigenvalue of multiplicity two. Setting $\sigma=\{-3 / 2,1 / 2\}$ and $\Sigma=\{-1 / 2,3 / 2\}$ one immediately concludes that in this case $d=\operatorname{dist}\{\sigma, \Sigma\}=1$ and the perturbation $V$ is off-diagonal with respect to the decomposition $\mathbb{C}^{4}=\operatorname{RanE}_{A}(\sigma) \oplus \operatorname{RanE}_{A}(\Sigma)$ and $\|V\|=\sqrt{3} d / 2$. However, $\mathcal{O}_{1 / 2}(\sigma)=(-2,-1) \cup(0,1)$ does not intersect the set $\operatorname{spec}(B)=\{-2,0,2\}$. 
Example 1.6. Let $\mathcal{H}=\mathbb{C}^{3}$,

$$
A=\left(\begin{array}{ccc}
-1 & 0 & 0 \\
0 & 0 & 0 \\
0 & 0 & 1
\end{array}\right) \quad \text { and } \quad V=\left(\begin{array}{ccc}
0 & \sqrt{2} & 0 \\
\sqrt{2} & 0 & 0 \\
0 & 0 & 0
\end{array}\right)
$$

The spectrum of the $3 \times 3$ matrix

$$
B=\left(\begin{array}{ccc}
-1 & \sqrt{2} & 0 \\
\sqrt{2} & 0 & 0 \\
0 & 0 & 1
\end{array}\right)
$$

consists of the two eigenvalues -2 and 1 , with 1 being an eigenvalue of multiplicity two. Setting $\sigma=\{0\}$ and $\Sigma=\{-1,1\}$ one concludes that $\mathcal{K}(\sigma) \cap \Sigma=\emptyset, d=$ $\operatorname{dist}\{\sigma, \Sigma\}=1$, the perturbation $V$ is off-diagonal with respect to the decomposition $\mathbb{C}^{4}=\operatorname{RanE}_{A}(\sigma) \oplus \operatorname{RanE}_{A}(\Sigma)$ and $\|V\|=\sqrt{2} d$. However, $\mathcal{O}_{1}(\sigma)=(-1,1)$ does not intersect the $\operatorname{set} \operatorname{spec}(B)=\{-2,1\}$.

\section{Perturbation of Spectral subspaces}

In this section we accomplish the proof of remaining statements of Theorem 1 part (ii) and Theorem 2 part (ii) related to the perturbation of spectral subspaces.

Proposition 2.1 ([6], [7, 17]). Let $A$ and $B$ be bounded self-adjoint operators and $\sigma$ and $\Delta$ two Borel sets on the real axis $\mathbb{R}$. Then

$$
\operatorname{dist}(\sigma, \Delta)\left\|\mathrm{E}_{A}(\sigma) \mathrm{E}_{B}(\Delta)\right\| \leq \frac{\pi}{2}\|A-B\| .
$$

If, in addition, the convex hull of the set $\sigma$ does not intersect the set $\Delta$, or the convex hull of the set $\Delta$ does not intersect the set $\sigma$, then one has the stronger result

$$
\operatorname{dist}(\sigma, \Delta)\left\|\mathrm{E}_{A}(\sigma) \mathrm{E}_{B}(\Delta)\right\| \leq\|A-B\| .
$$

The proof of Theorem 1 part (ii) is based on combining Proposition 2.1 with information on the shift of the spectrum obtained in Theorem 1 part (i).

Theorem 2.2. Assume Hypothesis 1.2, If

$$
\|V\|<\frac{3 \pi-\sqrt{\pi^{2}+32}}{\pi^{2}-4} d
$$

then

$$
\left\|\mathrm{E}_{A}(\sigma)-\mathrm{E}_{B}\left(\mathcal{O}_{d / 2}(\sigma)\right)\right\| \leq \frac{\pi}{2} \frac{\|V\|}{d-\delta_{V}}<1 .
$$

Proof. Introduce the notations $P=\mathrm{E}_{A}(\sigma)$ and $Q=\mathrm{E}_{A+V}\left(\mathcal{O}_{d / 2}(\sigma)\right)$. By Theorem 1.3 (i)

$$
Q^{\perp}=\mathrm{E}_{B}\left(\mathcal{U}_{\delta_{V}}(\Sigma)\right),
$$

where $\mathcal{U}_{\delta_{V}}(\Sigma)$ denotes the closed $\delta_{V}$-neighborhood of the set $\Sigma$.

By the first claim of Proposition 2.1

$$
\left\|P Q^{\perp}\right\| \leq \frac{\pi}{2} \frac{\|V\|}{\operatorname{dist}\left(\sigma, \mathcal{U}_{\delta_{V}}(\Sigma)\right)} .
$$

The distance between the set $\sigma$ and the (closed) $\delta_{V}$-neighborhood of the set $\Sigma$ can be estimated from below as follows

$$
\operatorname{dist}\left(\sigma, \mathcal{U}_{\delta_{V}}(\Sigma)\right) \geq d-\delta_{V}>0
$$


using the second claim of Theorem 1.3 . Then (2.2) implies the inequality

$$
\left\|P Q^{\perp}\right\| \leq \frac{\pi}{2} \frac{\|V\|}{d-\delta_{V}} .
$$

It is an elementary exercise to check that the function

$$
f(x)=\frac{\pi}{2} x+x \tan \left(\frac{1}{2} \arctan 2 x\right)-1
$$

strictly increases on the positive semi-axis and that $f(x)$ has a unique positive root $\frac{3 \pi-\sqrt{\pi^{2}+32}}{\pi^{2}-4}$. As a corollary, hypothesis (2.1) implies the inequality

$$
\frac{\pi}{2} \frac{\|V\|}{d-\delta_{V}}<1
$$

Hence,

$$
\left\|P Q^{\perp}\right\| \leq \frac{\pi}{2} \frac{\|V\|}{d-\delta_{V}}<1 .
$$

Interchanging the roles of $\sigma$ and $\Sigma$ one obtains the analogous inequality

$$
\left\|P^{\perp} Q\right\| \leq \frac{\pi}{2} \frac{\|V\|}{d-\delta_{V}}<1
$$

Since

$$
\|P-Q\|=\max \left\{\left\|P Q^{\perp}\right\|,\left\|P^{\perp} Q\right\|\right\}
$$

(see, e.g., 11, Ch. III, Section 39]), inequalities (2.4) and (2.5) prove the assertion.

We split the proof of Theorem 2 part (ii) into several steps.

1. First, we prove that the difference of the corresponding spectral projections is a strict contraction.

Lemma 2.3. Assume Hypothesis 1.2, If $\mathcal{K}(\sigma) \cap \Sigma=\emptyset$ and

$$
\|V\|<\sqrt{2} d
$$

then

$$
\left\|\mathrm{E}_{A}(\sigma)-\mathrm{E}_{B}\left(\mathcal{O}_{d}(\sigma)\right)\right\|<1 .
$$

Proof. Introduce the notations $P=\mathrm{E}_{A}(\sigma)$ and $Q=\mathrm{E}_{B}\left(\mathcal{O}_{d}(\sigma)\right)$. We also need to introduce four spectral projections associated with the operators $A$ and $B$ and let

$$
P_{\ell}=\mathrm{E}_{A}((-\infty, \inf \sigma-d]) \text { and } P_{r}=\mathrm{E}_{A}([\sup \sigma+d, \infty))
$$

and

$$
Q_{\ell}=\mathrm{E}_{B}((-\infty, \inf \sigma-d]) \text { and } Q_{r}=\mathrm{E}_{B}([\sup \sigma+d, \infty)) .
$$

Our first claim is that

$$
\left\|P_{k}-Q_{k}\right\|<\frac{\sqrt{2}}{2}, \quad k=\ell, r .
$$

It can be seen as follows. Since the perturbation $V$ is off-diagonal with respect to the decomposition $\mathcal{H}=\operatorname{RanE}_{A}(\sigma) \oplus \operatorname{RanE}_{A}(\Sigma)$, the operator $A+V$ can be 
represented as the following $3 \times 3$ Jacobi type operator-matrix with respect to the decomposition $\mathcal{H}=\operatorname{Ran} P_{\ell} \oplus \operatorname{Ran} P \oplus \operatorname{Ran} P_{r}$

$$
B=\left(\begin{array}{ccc}
A_{\ell} & V_{\ell \sigma} & 0 \\
V_{\sigma \ell} & A_{\sigma} & V_{\sigma r} \\
0 & V_{r \sigma} & A_{r}
\end{array}\right)
$$

Here we used the notation

$$
A_{k}=\left.A\right|_{\operatorname{Ran} P_{k}}, \quad k=\ell, r, \quad A_{\sigma}=\left.A\right|_{\operatorname{Ran} P}
$$

and

$$
V_{\sigma k}=\left.P V\right|_{\operatorname{Ran} P_{k}} \quad \text { and } \quad V_{k \sigma}=V_{k \sigma}^{*}, \quad k=\ell, r .
$$

The perturbation problem $A \longrightarrow B$ can naturally be split into two subproblems

$$
A=\left(\begin{array}{ccc}
A_{\ell} & 0 & 0 \\
0 & A_{\sigma} & 0 \\
0 & 0 & A_{r}
\end{array}\right) \longrightarrow \widetilde{A}=\left(\begin{array}{ccc}
A_{\ell} & 0 & 0 \\
0 & A_{\sigma} & V_{\sigma r} \\
0 & V_{r \sigma} & A_{r}
\end{array}\right) \longrightarrow B=\left(\begin{array}{ccc}
A_{\ell} & V_{\ell \sigma} & 0 \\
V_{\sigma \ell} & A_{\sigma} & V_{\sigma r} \\
0 & V_{r \sigma} & A_{r}
\end{array}\right) .
$$

The operator matrix $\widetilde{A}$ is block-diagonal with respect to the decomposition $\mathcal{H}=$ $\operatorname{Ran} P_{\ell} \oplus \operatorname{Ran} P_{\ell}^{\perp}$ and clearly $\|A-\widetilde{A}\|<\sqrt{2} d$. Applying Theorem 1.3(i, ii) to the "lower-dimensional" off-diagonal perturbation problem

$$
\left(\begin{array}{cc}
A_{\sigma} & 0 \\
0 & A_{r}
\end{array}\right) \longrightarrow\left(\begin{array}{cc}
A_{\sigma} & V_{\sigma r} \\
V_{r \sigma} & A_{r}
\end{array}\right)
$$

under hypothesis $\|V\|<\sqrt{2} d$ one concludes that the spectrum of $\widetilde{A}$ consists of two subordinated components, $\widetilde{\sigma}=\operatorname{spec}\left(A_{\ell}\right)=\Sigma \cap(-\infty, \inf \sigma-d]$ and "the remainder" $\widetilde{\Sigma}$. Moreover,

$$
\sup A_{\ell}=\sup \widetilde{\sigma}<\inf \sigma-\delta_{V} \leq \inf \widetilde{\Sigma}=\inf \left(\begin{array}{cc}
A_{\sigma} & V_{\sigma r} \\
V_{r \sigma} & A_{r}
\end{array}\right),
$$

where

$$
\delta_{V}=\|V\| \tan \left(\frac{1}{2} \arctan \frac{2\|V\|}{d}\right)<d .
$$

Applying Theorem 3 to the off-diagonal perturbation problem $\widetilde{A} \longrightarrow B$ where the spectra of the diagonal entries $A_{\ell}$ and $\left(\begin{array}{cc}A_{\sigma} & V_{\sigma r} \\ V_{r \sigma} & A_{r}\end{array}\right)$ are subordinated (cf. (2.9)) yields

$$
\left\|P_{\ell}-Q_{\ell}\right\|<\frac{\sqrt{2}}{2}
$$

Using analogous arguments one proves the remaining estimate

$$
\left\|P_{r}-Q_{r}\right\|<\frac{\sqrt{2}}{2} \text {. }
$$

Clearly,

$$
\left\|P^{\perp} Q\right\|=\left\|\left(P_{\ell}+P_{r}\right) Q\right\| \leq \sqrt{\left\|P_{\ell} Q\right\|^{2}+\left\|P_{r} Q\right\|^{2}}
$$

and moreover

$$
\begin{aligned}
& \left\|P_{\ell} Q\right\| \leq\left\|P_{\ell}\left(Q+Q_{r}\right)\right\|=\left\|P_{\ell} Q_{\ell}^{\perp}\right\| \leq\left\|P_{\ell}-Q_{\ell}\right\|, \\
& \left\|P_{r} Q\right\| \leq\left\|P_{r}\left(Q+Q_{\ell}\right)\right\|=\left\|P_{r} Q_{r}^{\perp}\right\| \leq\left\|P_{r}-Q_{r}\right\| .
\end{aligned}
$$

Thus,

$$
\left\|P^{\perp} Q\right\| \leq \sqrt{\left\|P_{\ell}-Q_{\ell}\right\|^{2}+\left\|P_{r}-Q_{r}\right\|^{2}}<1
$$


using (2.10) and (2.11). In an analogous way one proves that

$$
\left\|P Q^{\perp}\right\|<1
$$

and hence

$$
\|P-Q\|=\max \left\{\left\|P^{\perp} Q\right\|,\left\|P Q^{\perp}\right\|\right\}<1 .
$$

The proof is complete.

2. Next, we obtain the following general result which is of an a posteriori character.

Theorem 2.4. Assume Hypothesis 1.2. Suppose, in addition, that $\mathcal{K}(\sigma) \cap \Sigma=\emptyset$. Denote by $\mathcal{O}$ the maximal open (finite or semi-infinite) interval such that $\mathcal{O} \cap \Sigma=\emptyset$ and the spectrum $\widetilde{\sigma}$ of the operator $B$ in $\mathcal{O}$,

$$
\tilde{\sigma}=\operatorname{spec}(B) \cap \mathcal{O},
$$

is a closed set.

If

$$
\left\|\mathrm{E}_{A}(\sigma)-\mathrm{E}_{B}(\mathcal{O})\right\|<1
$$

then

$$
\left\|\mathrm{E}_{A}(\sigma)-\mathrm{E}_{B}(\mathcal{O})\right\| \leq \sin \arctan \left(\frac{\|V\|}{\operatorname{dist}(\widetilde{\sigma}, \Sigma)}\right)
$$

Proof. Introduce the notations $P=\mathrm{E}_{A}(\sigma)$ and $Q=\mathrm{E}_{B}(\mathcal{O})$. It is well known (see [14. Corollary 3.4]; cf. [5, Lemma 2.3], [8, Theorem 1], [11]) that if $\|P-Q\|<1$, then $\operatorname{Ran} Q$ is the graph of a bounded operator $X: \operatorname{Ran} P \rightarrow \operatorname{Ran} P^{\perp}$ and

$$
\|P-Q\|=\frac{\|X\|}{\sqrt{1+\|X\|^{2}}}
$$

Without loss of generality one may assume that

$$
\inf \widetilde{\sigma}=-\sup \widetilde{\sigma} .
$$

For any $g \in \operatorname{RanE}_{B}(\mathcal{O})$ one obtains that $P^{\perp} g=X P g$ and hence

$$
P^{\perp} B g=P^{\perp} B P g+P^{\perp} B P^{\perp} P^{\perp} g=P^{\perp} B P g+P^{\perp} B P^{\perp} X P g .
$$

Clearly the following estimates hold

$$
\left\|P^{\perp} B P g\right\|=\|V P g\| \leq\|V\|\|P g\|
$$

and

$$
(\sup \widetilde{\sigma}+\operatorname{dist}(\widetilde{\sigma}, \Sigma))\|X P g\| \leq\left\|P^{\perp} B P^{\perp} X P g\right\| .
$$

If in addition $g \in \operatorname{Ran} Q$ one obtains

$$
\begin{aligned}
\left\|P^{\perp} B g\right\| & =\left\|P^{\perp} Q B Q g\right\| \leq\left\|P^{\perp} Q\right\|\|Q B Q\|\|g\| \\
& \leq \frac{\|X\|}{\sqrt{1+\|X\|^{2}}} \sup \widetilde{\sigma} \sqrt{1+\|X\|^{2}}\|P g\|=\sup \widetilde{\sigma}\|X\|\|P g\| .
\end{aligned}
$$

Combining (2.15)-(2.17), and (2.18) one gets the inequality

$$
(\sup \widetilde{\sigma}+\operatorname{dist}(\widetilde{\sigma}, \Sigma))\|X P g\| \leq(\sup \widetilde{\sigma}\|X\|+\|V\|)\|P g\|, \quad g \in \operatorname{Ran} Q .
$$


Since (2.19) holds for any $g \in \operatorname{Ran} Q$, one concludes that

$$
\|X\| \leq \frac{\|V\|}{\operatorname{dist}(\widetilde{\sigma}, \Sigma)}
$$

which proves the assertion in view of (2.14).

Remark 2.5. Assertion (2.13) is equivalent to the estimate

$$
\|\tan \Theta\| \leq \frac{\|V\|}{\operatorname{dist}(\widetilde{\sigma}, \Sigma)}
$$

where $\Theta$ is the operator angle between the subspaces $\operatorname{RanE}_{A}(\sigma)$ and $\operatorname{RanE}_{B}(\mathcal{O})$. (For discussion of this notion see, e.g., [14]). Thus, Theorem 2.4 is a generalization of the Davis-Kahan $\tan \Theta$-Theorem which is one from four fundamental estimates on the norm of the difference of spectral projections known as $\sin \Theta, \sin 2 \Theta, \tan \Theta$, and $\tan 2 \Theta$ Theorems proved by Davis and Kahan in [9] and [10].

3. Finally, rough estimate (2.8) of Lemma 2.3 can be sharpened by a posteriori result of Theorem 2.4 in combination with the result of Theorem 2 part (i). The proof of Theorem 2 part (ii) is as follows.

Theorem 2.6. Assume Hypothesis 1.2. If $\mathcal{K}(\sigma) \cap \Sigma=\emptyset$ and

$$
\|V\|<\sqrt{2} d
$$

then

$$
\left\|\mathrm{E}_{A}(\sigma)-\mathrm{E}_{B}\left(\mathcal{O}_{d}(\sigma)\right)\right\| \leq \sin \arctan \left(\frac{\|V\|}{d-\delta_{V}}\right)<1
$$

where

$$
\delta_{V}=\|V\| \tan \left(\frac{1}{2} \arctan \frac{2\|V\|}{d}\right) .
$$

Proof. By Theorem 2 part (i) and Lemma 2.3 the set $\mathcal{O}=\mathcal{O}_{d}(\sigma)$ satisfies the hypothesis of Theorem 2.4 with

$$
\operatorname{dist}(\widetilde{\sigma}, \Sigma)=d-\delta_{V}
$$

Applying Theorem 2.4 completes the proof.

\section{ACKNOWLEDGMENTS}

A. K. Motovilov acknowledges the kind hospitality and financial support by the Department of Mathematics, University of Missouri, Columbia, MO, USA. He was also supported in part by the Deutsche Forschungsgemeinschaft and the Russian Foundation for Basic Research.

\section{REFERENCES}

1. N. I. Achiezer and I. M. Glasmann, Theory of Linear Operators in Hilbert Space, Dover Publications, New York, 1993. MR.1255973 (94i:47001)

2. V. Adamyan and H. Langer, Spectral properties of a class of rational operator valued functions, J. Operator Theory 33 (1995), 259 - 277. MR1354980 (96i:47023)

3. V. Adamyan, H. Langer, and C. Tretter, Existence and uniqueness of contractive solutions of some Riccati equations, J. Funct. Anal. 179 (2001), 448 - 473. MR1809118 (2001j:34074)

4. S. Albeverio, K. A. Makarov, and A. K. Motovilov, Graph subspaces and the spectral shift function, Canad. J. Math. 55 (2003), 449 - 503. MR1980611(2004d:47031)

5. C. Apostol, C. Foias, and N. Salinas, On stable invariant subspaces, Integr. Equat. Oper. Theory 8 (1985), 721 - 750. MR0818331(87c:47005) 
6. R. Bhatia, C. Davis, and P. Koosis, An extremal problem in Fourier analysis with applications to operator theory, J. Funct. Anal. 82 (1989), 138 - 150. MR0976316 (91a:42006)

7. R. Bhatia, C. Davis, and A. McIntosh, Perturbation of spectral subspaces and solution of linear operator equations, Linear Algebra Appl. 52/53 (1983), 45 - 67. MR0709344 (85a:47020)

8. J. Daughtry, Isolated solutions of quadratic matrix equations, Linear Algebra Appl. 21 (1978), 89 - 94. MR0485926 (58:5720)

9. C. Davis, The rotation of eigenvectors by a perturbation. I and II, J. Math. Anal. Appl. 6 (1963), 159 - 173; 11 (1965), 20 - 27. MR0149309 (26:6799) MR0180852(31:5082)

10. C. Davis and W. M. Kahan, The rotation of eigenvectors by a perturbation. III, SIAM J. Numer. Anal. 7 (1970), 1 - 46. MR0264450(41:9044)

11. P. R. Halmos, Two subspaces, Trans. Amer. Math. Soc. 144 (1969), 381-389. MR0251519 (40:4746)

12. T. Kato, Perturbation Theory for Linear Operators, Springer-Verlag, Berlin, 1966. MR0203473 (34:3324)

13. V. Kostrykin, K. A. Makarov, and A. K. Motovilov, On a subspace perturbation problem, Proc. Amer. Math. Soc. 131 (2003), 3469 - 3476. MR1991758 (2004c:47029)

14. V. Kostrykin, K. A. Makarov, and A. K. Motovilov, Existence and uniqueness of solutions to the operator Riccati eqution. A geometric approach, in Yu. Karpeshina, G. Stolz, R. Weikard, Y. Zeng (Eds.), Advances in Differential Equations and Mathematical Physics, Contemporary Mathematics 327, Amer. Math. Soc., 2003, p. 181 - 198. MR.1991541 (2004f:47012)

15. V. Kostrykin, K. A. Makarov, and A. K. Motovilov, A generalization of the $\tan 2 \Theta$ theorem, in J. A. Ball, M. Klaus, J. W. Helton, and L. Rodman (Eds.), Current Trends in Operator Theory and Its Applications, Operator Theory: Advances and Applications Vol. 149. Birkhäuser, Basel, 2004, p. 349 - 372. MR2063758 (2005d:47041)

16. H. Langer, A. Markus, V. Matsaev, and C. Tretter, A new concept for block operator matrices: The quadratic numerical range, Linear Algebra Appl. 330 (2001), 89 - 112. MR1826651 (2002b:47015)

17. R. McEachin, Closing the gap in a subspace perturbation bound, Linear Algebra Appl. 180 (1993), 7 - 15. MR.1206407 (94c:47017)

Fraunhofer-Institut für Lasertechnik, Steinbachstrasse 15, D-52074 Aachen, GerMANY

E-mail address: kostrykin@ilt.fraunhofer.de

Current address: Institut für Mathematik, Technische Universität Clausthal, Erzstraße 1, D38678 Clausthal-Zellerfeld, Germany

E-mail address: kostrykin@t-online.de

Department of Mathematics, University of Missouri, Columbia, Missouri 65211

E-mail address: makarov@math.missouri.edu

Department of Mathematics, University of Missouri, Columbia, Missouri 65211

Current address: Bogoliubov Laboratory of Theoretical Physics, JINR, 141980 Dubna, Moscow Region, Russia

E-mail address: motovilv@thsun1.jinr.ru 